Special Issue: Social Cognition and Self. Citation: Moore, K. (2015). Rumination and self-destructive thoughts in people with depression. Behavioural Sciences Undergraduate Journal, 2(1), 5-12.

Author: Kelsie Moore, Psychology, Mount Royal University (MRU)

Correspondence: kmoor481@mtroyal.ca

Reviewers: Alain Morin, MRU; Christina Campbell, MRU; Kyle Gardiner, University of Calgary; Nancy Ogden, MRU

Editor: Famira Racy, MRU

Acknowledgements: The BSUJ thanks Dr. Dave Mumby from Concordia University for Associate Editorial contribution and Dr. Alain Morin for Guest Editorial research guidance.

Author Acknowledgements: Kelsie Moore thanks Dr. Alain Morin for his guidance and encouragement on getting this article published, as well as the ongoing support of her family.

Copyright: (C) 2015 Kelsie Moore. This is an open-access article distributed under the terms of the Creative Commons Attribution 4.0 International License. The use, distribution or reproduction of this article in other forums is permitted, provided the original author(s) or licensor are credited, a link to the licence is provided (CC for 4.0), it is indicated whether or not changes were made to the original article, and other conditions of the BSUJ and the other forums are met.

\title{
Rumination and Self-destructive Thoughts in People with Depression
}

\begin{abstract}
Increasing rates of suicide have peaked interest in how individuals with depression may view their environment differently and have abnormal thought processes compared to non-depressed individuals. In order to explore how depressive thought processes lead to completed suicide, this review of literature looks at research and theoretical explanations of rumination, self-destructive thoughts, and feelings of hopelessness. People with depression are more attentive to both their own and other people's actions and have a strong desire for control. Rumination prevents effective problem solving and increases the duration of depressive symptoms. Highly self-aware individuals often attend to discrepancies between the real self and who they wish to be, which causes distress and overthinking about negative aspects of problems while neglecting the development of solutions. Suicide is one method of escaping the self when these discrepancies become salient and continual failure also leads to feelings of hopelessness. Positive distraction such as getting involved in hobbies as well as the promotion of optimistic and hopeful thoughts may help depressive ruminators refrain from negative thoughts and ultimately help prevent suicide.
\end{abstract}

Keywords: self, self-awareness, goal-setting, worry, rumination, depression, hopelessness, selfdestruction, suicide

\section{Introduction}

Within the realm of psychological research, there has been a large amount of interest in how thought processes differ in people with depression compared to non-depressed individuals. Depressed people think differently about themselves and others in their social world and this paper will address how these people tend to make more social inferences in order to understand their own and other people's behaviour (Gleicher \& Weary, 1991). Depressed individuals pay closer attention to their own and other's actions in an effort to better understand reasons for human behaviour. Ability to predict and understand outcomes helps these individuals gain a sense of control. This type of thinking may seem adaptive, but in reality it can result in self- 
destructive thoughts, uncertainty about one's environment, and feelings of hopelessness (NolenHoeksema, Wisco, \& Lyubomirsky, 2008). Depressed individuals often overthink or ruminate about their problems and distressing feelings. This can lead to the development and increased accessibility to self-destructive thoughts that can ultimately lead to suicide (Chatard \& Selimbegović, 2011). In this paper I explore research on the relationships between rumination, depression, and self-destructive thoughts. I review recent literature in order to critically analyze the relationship between depression and maladjusted thought processes by defining the main concepts of rumination and depression and exploring the various theories and measures applied in psychological research.

\section{Rumination}

In the literature rumination is defined as a way of thinking which involves repetitive, fixated thoughts that analyze problems and associated distress with less attention focussed on solutions (Moulds, Kandris \& Wong, 2007; Nolen-Hoeksema et al., 2008). Scholars explain that rumination consists of both a brooding and a reflection component in which brooding involves repetitive dwelling on unfavorable outcomes whereas reflection involves information-seeking to better understand one's distress (Tucker, Wingate, O’Keefe, Mills, Rasmussen, Davidson, \& Grant, 2013). Nolen-Hoeksema et al. (2008) explained that participants prone to rumination often claimed they were trying to make sense of their problems in order to come up with solutions, when in reality rumination may have hindered the likelihood of coming up with plausible solutions and actually implementing those solutions. Further, ruminators regularly procrastinated and wanted more time to work on solutions to complex problems before they committed to them. Also, participants showed less confidence in their solutions and worried about obstacles when trying to solve problems (Nolen-Hoeksema et al., 2008).

\subsection{Worry and Rumination}

Rumination is significantly correlated with worry but there are distinguishing factors between the two concepts (Nolen-Hoeksema et al., 2008). Although both forms of thought are self-focused, repetitive, abstract, and inflexible, worry is more future-oriented whereas rumination often involves thinking about past mistakes and fixating on why those mistakes occurred. Rumination is more avoidant and can lead people to believe that outcomes are uncontrollable. Studies suggest that this may actually be helpful in limiting the impact of negative information on the individual, while people who worry know that they have some control over the outcomes as long as they make an effort (Moulds et al., 2007; Nolen-Hoeksema et al., 2008). Encouraging those who ruminate to disengage from their negative thoughts and direct their attention towards pleasant activities to act as positive distractions is a plausible solution to rumination (Nolen-Hoeksema et al., 2008). Positive distractions may include going for a run or watching a movie with friends whereas negative distractions include reckless driving, excessive drinking, or drug abuse. Getting involved in enjoyable positive activities may work for some individuals, but others may find it hard to discover an activity that will truly interest them enough to take their mind off of their distressing thoughts. Those who ruminate may try to engage in many activities desperately trying to distract themselves from their problems, but still they find no relief (Moulds, Kandris, Starr, \& Wong, 2007; Nolen-Hoeksema et al., 2008). More research needs to be done on this issue in order to find a positive solution, however individual differences can render it difficult to find a solution that would work for everyone. 


\subsection{Self-reflection, Self-rumination and Measures}

Rumination is strongly associated with depressive symptoms and can be used as a predictive factor for developing depressive disorders (Moulds et al., 2007), while self-reflection is a healthier form of self-focused attention (Takano \& Tanno, 2009). Some researchers addressed the differences between the two subtypes of self-focused attention (self-reflection and self-rumination) hypothesizing that self-reflection would have adaptive properties whereas selfrumination would be associated with maladaptive properties. The adaptive outcomes of selffocused attention include self-regulation, problem solving and increased self-knowledge (Takano \& Tanno, 2009). In order to assess both subtypes, Takano and Tanno (2009) administered a Rumination-Reflection Questionnaire which has been shown to have acceptable test-retest reliability and convergent validity. This questionnaire assesses participants' tendency to either ruminate or self-reflect by asking questions about how often they engage in 22 ruminative or reflective thoughts or behaviours when they are feeling depressed or sad on a 5-point scale (Nolen-Hoeksema et al., 2008; Takano \& Tanno, 2009). Takano and Tanno (2009) discovered that self-reflection was motivated by curiosity and was linked with openness to experience and may be useful in problem solving and depressed mood regulation in the absence of rumination. On the other hand, they found that self-rumination was motivated by perceived threats and was linked to neuroticism and depression.

Another similar measure of rumination is the Ruminative-Response Questionnaire, also comprised of 22 items that measure rumination as well as two five-question subscales on brooding and reflection (Tucker et al., 2013). Brooding is a term used to describe people's focus on their own depressed mood and its consequences, whereas reflection is described as the attempt to understand why one is in a depressed state (Tucker et al., 2013).

\section{Depression}

People with depression have unique ways of thinking about themselves and others in their environment. Donaldson, Lam, and Mathews (2007) found evidence regarding attentional biases for negative information in major depression and this was also linked to rumination. Since Takano and Tanno (2009) confirmed that rumination and negative self-focus were weaknesses that could lead to the development of depression, this relationship has been a common focus of many other works in psychological research. Moulds et al. (2007) defined depression as a dysfunctional disorder that involves avoidance, withdrawal, and inactivity in one's social environment. It is said that $13-19 \%$ of people are diagnosed with depression and about $80 \%$ of first-episode patients experience relapse (Huffziger \& Kuehner, 2009).

Several studies use a common measure of depression called the Beck Depression Inventory which consists of 21 self-report questions that measure symptoms and severity of depression in clinical and non-clinical populations (Gleicher \& Weary, 1991; Jones, Papadakis, Hogan, \& Strauman, 2009; Moulds et al., 2007). Another measure of depression mentioned in the literature that has high internal consistency is the Self-rating Depression Scale which has 20 self-report questions measuring the psychological and physical symptoms of depression (Dean \& Range, 1999). If researchers wish to assess recent symptoms of depression, the Center for Epidemiologic Studies Depression Scale includes 20 questions measured on a 0-3 Likert-scale on symptoms experienced within the past two weeks (Tucker et al., 2013). 


\subsection{Depression and Rumination}

Ruminative thought and depression often go hand in hand. A common theory addressed in much of the research is response-styles theory which explains when depressed individuals ruminate about their distressing symptoms, they likely remain depressed for longer and their depression becomes more severe (Moulds et al., 2007). Response-styles theory views rumination as a stable trend, focusing on the self in response to depressed mood (Watkins \& NolenHoeksema, 2014). This theory also states the duration and severity of depression depends on how the individual responds to his/her environment (Moulds et al., 2007). In their studies, Gleicher and Weary (1991) found that compared to non-depressed people, individuals with depression were more motivated to understand, predict, and control their social environment as well as others' social behaviour. Despite these aspirations to understand their surroundings, depressed people reported less confidence in achievement and felt they had less control over their personal outcomes (Gleicher \& Weary, 1991).

Gleicher and Weary (1991) also learned that depressed participants were more vigilant than non-depressed participants when it came to information processing, and they generated more abstract social inferences but felt less confident in their overall impressions and future predictions of other's behaviour. Chatard and Selimbegović (2011) found that even though participants experienced a loss of control following failure, the majority were motivated to escape from negative self-awareness and there was no evidence to support the idea that they would try to restore their sense of control.

Authors claim that rumination is reinforced by avoidance so that the impact of distressing, difficult situations is reduced and it can also be reinforced by others (Moulds et al., 2007). For example, Nolen-Hoeksema et al. (2008) say that it is reinforcing when a depressed person's negative self-concept is verified by other people. Furthermore, depressed people attend to and believe feedback from others that confirms their own negative self view (NolenHoeksema et al., 2008). Depression can develop into major depression if chronic ruminators start to lose social support which is said to be a common occurrence (Nolen-Hoeksema et al., 2008).

\section{Goal Setting and Control Theory}

Response-styles theory researchers report that depressive rumination enhances negative mood and interferes with problem solving and goal setting (Watkins \& Nolen-Hoeksema, 2014). On the other hand, researchers in the field of self-regulation and control theory hypothesize that episodes of ruminative thought originate from discrepancies between one's current situation and one's desired goals and this continues until the goal is either reached or rejected (NolenHoeksema et al., 2008; Watkins \& Nolen-Hoeksema, 2014). Goal-oriented rumination can help if the person attends to the goal in order to achieve it rather than only focusing on those discrepancies. However, this is not often the case with ruminators because these people tend to ruminate in order to avoid actively solving their problems or working towards their goals (Watkins \& Nolen-Hoeksema, 2014).

Jones et al., (2009) found that when individuals were unable to effectively pursue their goals, they generated more depressive symptoms, especially when they scored high on rumination in relation to self-reflection. Jones and colleagues further explained that continued failure leads to increased negative self-evaluation and people become less motivated to accomplish their goals because they develop a sense of hopelessness. Not all people who fail to attain their goals become depressed, but Jones et al. (2009) found that high levels of goal failure, 
high levels of rumination, and low levels of reflection were correlated with depressive symptoms after controlling for other contributing factors.

\subsection{Self-awareness and Related Theories}

Self-awareness is defined as attention toward the self (Chatard \& Selimbegović, 2011). Self-awareness can be informed by comparison of the real and ideal self (e.g., who and I?' versus 'who do I want to be?') and discrepancies between these views of the self leads to anxiety from which the self tries to reconcile or escape . Selimbegović and Chatard (2013) illustrated that increased aversive self-awareness influences self-destructive thoughts. Aversive self-awareness occurs when people have increased focus on the self and they focus on aspects that they dislike which can lead to negative feelings and thoughts of escaping the self by (e.g., thoughts of putting themselves in harm's way). Studies have shown that when people fail to attain certain important standards or goals, they may try to escape negative self-awareness which can lead to suicidal ideation (Chatard \& Selimbegović, 2011).

Objective self-awareness theory describes that a discrepancy between the real self and the ideal self results in an aversive state of self-awareness which makes self-evaluation more negative and can produce a sense of failure, therefore Selimbegović and Chatard (2013) sought to measure this. The researchers induced self-awareness by exposing participants to a mirror while doing a lexical decision task and they found that compared to controls, the participants in the mirror exposure condition recognized suicide-related words faster than neutral words, suggesting more access to more negative self-evaluations and related suicidal thoughts.

Escape theory, another theory commonly found in the literature, suggests that attempted suicide is the result of trying to escape from a painful state of self-awareness, rather than actual desire to die (Chatard \& Selimbegović, 2011; Selimbegović \& Chatard, 2013). This theory describes six main stages that lead to suicide which include falling short of standards resulting in major disappointment, attributions of the self (often self-blame), high self-awareness, negative affect, cognitive deconstruction, and consequences of deconstruction such as disinhibition, absence of emotions and irrationality which would make suicidal thoughts more appealing and less scary (Dean \& Range, 1999; Chatard \& Selimbegović, 2011). Cognitive deconstruction is explained as a subjective shift towards negative affect in which the individual produces less integrative and meaningful thoughts and awareness (Dean \& Range, 1999). Chatard and Selimbegović (2011) based their hypotheses on escape theory in that failure to attain important standards motivates individuals to escape their negative self-awareness and therefore increases the accessibility of suicidal thoughts. They discovered that the larger the discrepancy between the actual self and their ideal standards, the more likely people will think of suicide and concepts related to escape following failure. Chatard and Selimbegović's (2011) studies propose that holding high standards and not living up to them can be detrimental to an individual and can make access to suicidal thoughts easier. Dean and Range (1999) add that the consequence of high expectations can be even more severe with added stress.

\section{Self-destructive Thoughts}

Since there is a strong relationship between rumination and depression, Morrison and O’Connor (2008) explored whether rumination could be a precursor for suicidal behaviour since it is also linked to impaired problem solving which is commonly observed in suicidal individuals. In their meta-analysis they found that rumination was significantly associated with thoughts and/or behaviour consistent with suicidal tendencies and was able to predict suicidal 
ideation despite various methodologies, samples, and measures. Suicidal ideation involves thinking about killing oneself and behaviour could be planning or attempting physical harm to oneself.

Self-destructive thoughts are often the result of major depression in which the person begins to consider ways to escape from their negative moods and failures. When depressed individuals fail to attain their goals, their thought patterns may become increasingly negative and may begin to be more self-destructive in nature. It is important to look at what types of situations generate these self-destructive and suicidal thoughts because according to Chatard and Selimbegović (2011), rates of suicide are increasing world-wide and account for about one million deaths per year. Since these initial thoughts are precursors to completed suicide, it is important to explore how and why individuals develop suicidal thoughts in order to better predict and prevent suicides from happening.

\subsection{Self-destructive Thoughts and Hopelessness}

Negative evaluations of the self lead to feelings of depression and hopelessness. Scholars suggest that feelings of hopelessness increase the likelihood that ruminative thoughts result in suicidal ideation; thus, hopelessness acts as a mediator between rumination and depressive symptoms that lead to self-destructive thoughts (Morrison \& O'Connor, 2008; Tucker et al., 2013). Tucker et al. (2013) introduced hope theory which explains that hope motivates people to find the right path to achieve their goals. Dean and Range (1999) describe that hopeless individuals often expect negative outcomes and they are unable to predict a pleasant future. They report that hopelessness is a better predictor of suicidal behaviour than depression. Hopelessness can be measured by the Hopelessness Scale, which consists of 20 true-false questions regarding future expectations (Dean \& Range, 1999). This measure is correlated with measures of future orientation, depression, and pessimism (Dean \& Range, 1999). Tucker et al. (2013) hypothesized that higher levels of hope and optimism would decrease levels of rumination and suicidal ideation and results confirmed this idea with significant correlations even when controlling for symptoms of depression. Therefore, hope and optimism provided protection for those ruminators (Tucker et al., 2013).

\section{Future Research}

Future research should focus on discovering ways to increase optimism and hope in depressed individuals since it can mean the difference between suicide and developing future goals. Watkins and Nolen-Hoeksema (2014) argued that interventions for depression should strive towards reducing rumination habits because patients will relapse otherwise. NolenHoeksema et al. (2008) suggest finding ways to help ruminators take their minds off of their negative thoughts such as getting involved in positive hobbies and activities. However, it would be interesting to find out if engaging in positive activities would simply foster avoidance instead of directing problem solving. Another potential avenue of research would consider sex differences in relation to suicide because some studies have suggested that women are more likely to ruminate than men (Morrison \& O'Connor, 2008; Nolen-Hoeksema et al., 2008). Also, much of the research focussed on non-clinical populations, which could limit generalization and those studies, should be replicated with clinical populations in the event that results differ. Furthermore, authors suggest that future research should look at levels of self-esteem, close relationships, and different cultural beliefs and values and how they may influence suicidal thoughts (Chatard \& Selimbegović, 2011). 


\section{Conclusion}

Since suicide rates are increasing, it is important to investigate influential factors such as depression and ruminative thoughts that lead to self-destructive thoughts that could eventually result in completed suicide. This review of literature outlined the concepts of rumination, depression, and self-destructive thoughts and provided theoretical explanations and common measures used in research. Studying rumination is essential to psychological research because rumination involves excessive thinking about one's problems, which interferes with one developing and implementing helpful solutions.

This paper outlined the differences between types of worry including future-oriented thoughts and the past-oriented thoughts involved in rumination. A plausible way to treat rumination might be to find positive distractions to help disengage from negative thoughts. Moulds et al. (2007) argued that rumination is highly related to depression and ruminators often experience severe depressive symptoms for a longer duration. People with depression are described as determined to understand, control, and predict their environment even though they lack confidence (Gleicher \& Weary, 1991).

Theorists argue for conceptual underpinnings of rumination, depression, and suicide. Control theory says that depression escalates if there are large discrepancies between the current situation and one s goals and depressed people often become hopeless when they continue to fail (Watkins \& Nolen-Hoeksema, 2014). As escape theory indicates, this leads to self-destructive thoughts where the individual tries to escape their negative emotions and aversive selfawareness. However, there is some evidence to support the arguments that increased optimism and hope mediate the effects of ruminative depression and protect individuals from suicidal thoughts in the long-term, and positive disengagement strategies may facilitate coping in the short-term.

Future researchers could investigate whether engaging in positive activities would simply foster avoidance instead of facilitating directing problem solving in everyday events. Further, more research is needed to consider sex differences in relation to both suicide and rumination. It is also unclear to what extent rumination exists in non-clinical samples, if at all. Intrapersonal and interpersonal processes such as self-esteem and close relationships should also be studied because these may also vary across individuals and may affect all of rumination, depression, and suicide.

\section{References}

Chatard, A., \& Selimbegović, L. (2011). When self-destructive thoughts flash through the mind: Failure to meet standards affects the accessibility of suicide-related thoughts. Journal of Personality and Social Psychology, 100(4), 587-605. doi:10.1037/a0022461

Dean, P. J., \& Range, L. M. (1999). Testing the escape theory of suicide in an outpatient clinical population. Cognitive Therapy and Research, 23(6), 561-572. doi:10.1023/A:1018728606568

Donaldson, C., Lam, D., \& Mathews, A. (2007). Rumination and attention in major depression. Behaviour Research and Therapy, 45(11), 2664-2678. doi:10.1016/j.brat.2007.07.002

Gleicher, F., \& Weary, G. (1991). Effect of depression on quantity and quality of social inferences. Journal of Personality and Social Psychology, 61(1), 105-114. doi:10.1037/00223514.61.1.105

Huffziger, S., \& Kuehner, C. (2009). Rumination, distraction, and mindful self-focus in depressed patients. Behaviour Research and Therapy, 47(3), 224-230. doi:10.1016/j.brat.2008.12.005

Jones, N. P., Papadakis, A. A., Hogan, C. M., \& Strauman, T. J. (2009). Over and over again: Rumination, reflection, and promotion goal failure and their interactive effects on depressive symptoms. Behaviour Research and Therapy, 47(3), 254-259. doi:10.1016/j.brat.2008.12.007 
Morrison, R., \& O'Connor, R. C. (2008). A systematic review of the relationship between rumination and suicidality. Suicide \& Life-Threatening Behavior, 38(5), 523-538.

Moulds, M. L., Kandris, E., Starr, S., \& Wong, A. C. (2007). The relationship between rumination, avoidance and depression in a non-clinical sample. Behaviour Research and Therapy, 45(2), 251261. doi:10.1016/j.brat.2006.03.003

Nolen-Hoeksema, S., Wisco, B. E., \& Lyubomirsky, S. (2008). Rethinking rumination. Perspectives on Psychological Science, 3(5), 400-424. doi:10.1111/j.1745-6924.2008.00088.x

Selimbegović, L., \& Chatard, A. (2013). The mirror effect: Self-awareness alone increases suicide thought accessibility. Consciousness and Cognition: An International Journal, 22(3), 756-764. doi:10.1016/j.concog.2013.04.014

Takano, K., \& Tanno, Y. (2009). Self-rumination, self-reflection, and depression: Self-rumination counteracts the adaptive effect of self-reflection. Behaviour Research and Therapy, 47(3), 260264. doi:10.1016/j.brat.2008.12.008

Tucker, R. P., Wingate, L. R., O’Keefe, V. M., Mills, A. C., Rasmussen, K., Davidson, C. L., \& Grant, D. M. (2013). Rumination and suicidal ideation: The moderating roles of hope and optimism. Personality \& Individual Differences, 55(5), 606-611. doi:10.1016/j.paid.2013.05.013

Watkins, E. R., \& Nolen-Hoeksema, S. (2014). A habit-goal framework of depressive rumination. Journal of Abnormal Psychology, 123(1), 24-34. doi:10.1037/a0035540 